\title{
MOVPE growth and characterization of Ga(NAsP) laser structures monolithically integrated on $\mathrm{Si}(001)$ substrates
}

\author{
S. Liebich, M. Zimprich, P. Ludewig, A. Beyer, K. Volz, W. Stolz \\ Structure and Technology Research Laboratory (STRL) and Department of Physics, Philipps-University, \\ Hans-Meerwein-Strasse, 35032 Marburg, Germany \\ B. Kunert \\ NAsP III/V GmbH, Knechtacker 19, 35041 Marburg, Germany \\ N. Hossain, S.R. Jin, S.J. Sweeney \\ Advanced Technology Institute and Department of Physics, University of Surrey, \\ Guildford, Surrey GU2 7XH, UK
}

\begin{abstract}
Laser structures containing the dilute nitride material $\mathrm{Ga}(\mathrm{NAsP})$ can be grown lattice matched on silicon substrates with high crystal quality and low defect density. Lasing operation from broad area lasers up to $120 \mathrm{~K}$ is verified.
\end{abstract}

The implementation of an efficient light source on silicon is the key technology for optical data communication on chip level. Our approach to realize CMOS-compatible laser structures on $\mathrm{Si}$ is the monolithic integration of the novel direct band gap material $\mathrm{Ga}(\mathrm{NAsP})$ which can be grown lattice matched on $\mathrm{Si}$ [1]. Its unique band structure can be explained in the framework of the band anticrossing model (BAC) [2, 3]. Lasing from a Ga(NAsP) laser on GaP under electrical injection was previously demonstrated [4]. The integration of the laser material on $\mathrm{Si}$ is more crucial. In particular, the defect-free nucleation of $\mathrm{GaP}$ on $\mathrm{Si}$ is essential to allow the growth of the active material and thick laser waveguide/barrier layers of $(\mathrm{BGa}) \mathrm{P}$ or $(\mathrm{BGa})(\mathrm{AsP})$ without introducing defects and cracking. In this work we focus on the MOVPE growth of Ga(NAsP) laser structures for electrical current injection lattice matched on exactly orientated Si substrates and their structural characterization.

The MOVPE growth took place in a commercially available Aixtron AIX 200-GFR reactor system at a reduced pressure of 50 mbar using Pd-purified $\mathrm{H}_{2}$ as carrier gas. The laser structures were grown at a reactor temperature of $575^{\circ} \mathrm{C}$ applying all-liquid precursors. Tertiarybutyl phosphine (TBP), tertiarybutyl arsine (TBAs), unsymmetric dimethyl hydrazine (UDMHy), triethyl gallium (TEGa) and triethyl boron (TEB) were used as sources for P, As, N, Ga and B, respectively. For the doping of the contact layers diethyl zinc (DEZn) and diethyl tellurium (DETe) were chosen as sources for $\mathrm{Zn}$ and Te as p- and n-dopants, respectively. Fig. 1 shows a schematic of the realized multi-quantum well (QW) laser structure.

The first step for defect-free III/V integration on exactly oriented Si (001) is the nucleation of GaP. Due to the different crystal structure of Si and III/V compound materials a double-stepped Si surface is necessary which can be achieved under special annealing conditions [5]. The nucleation of $3 \mathrm{~nm} \mathrm{GaP}$ at a low temperature of $450^{\circ} \mathrm{C}$ and an overgrowth with $55 \mathrm{~nm} \mathrm{GaP}$ at a higher temperature of $675^{\circ} \mathrm{C}$ allows for defect-free III/V growth and the self-annihilation of any formed anti-phase domains within the GaP crystal after a few tens of nanometers, respectively.

Because of the different thermal expansion coefficients of $\mathrm{Si}$ and the III/V material, the (BGa)P contact layers must be lattice matched at the growth temperature to avoid initialization of defects, which is realized by the incorporation of about 3\% B. Precise overall strain-management allows the growth of a several- $\mu \mathrm{m}$-thick III/V device structure without cracking. Doping levels of the p- and ncontacts, which is also crucial for optimal current injection, were adjusted via Hall measurements and secondary ion mass spectroscopy (SIMS) on test samples on GaP and Si substrates, respectively.

The active material $\mathrm{Ga}\left(\mathrm{N}_{\sim 7 \%} \mathrm{As}_{\sim 80 \%} \mathrm{P}\right)$ of the $3 \mathrm{QW}$ structure is surrounded by $3 \mathrm{~nm}$ thick GaP layers to prevent the formation of strong $\mathrm{B}-\mathrm{N}$ bonds and $20 \mathrm{~nm}$ thick $\left(\mathrm{B}_{5.5 \%} \mathrm{Ga}\right)\left(\mathrm{As}_{11 \%} \mathrm{P}\right)$ barriers for strain compensation of the highly compressively strained QW material. This active region is embedded in a separate confinement heterostructure $(\mathrm{SCH})$ consisting of a $100 \mathrm{~nm}(\mathrm{BGa})(\mathrm{AsP})$ linear grading and $50 \mathrm{~nm}\left(\mathrm{~B}_{5.5 \%} \mathrm{Ga}\right)\left(\mathrm{As}_{11 \%} \mathrm{P}\right)$. 
The QW-photoluminescence (PL) can be increased by applying optimal post-growth annealing conditions to the sample. Investigations of the PL intensity and line width show a clear dependency on annealing temperature, time and also on the composition of the SCH and barrier material, as will be discussed. For structural investigations high-resolution X-ray diffraction (XRD) around the (004) reflection of Si were performed together with dynamical simulation of this XRD pattern (fig. 3). The well-resolved satellite peaks even at high diffraction angles and the good agreement of measurement and simulation indicate sharp interfaces within the structure and the high crystal quality of this III/V laser on Si.

Additional transmission electron microscopy and SIMS analysis validate the defect-free growth and the well-adjusted doping profile, respectively, and will be discussed in more detail at the conference. From this structure broad area laser were fabricated. The light emission as a function of applied current for different temperatures shows a clear threshold behavior (fig. 4) verifying lasing operation under electrical injection. Lasing up to $120 \mathrm{~K}$ with a threshold current density $\mathrm{J}_{\text {th }}$ per $\mathrm{QW}$ of $1.47 \mathrm{kA} / \mathrm{cm}^{2}$ and an emission wavelength of about $835 \mathrm{~nm}$ could be observed.

For the first time electrically pumped lasing from $\mathrm{Ga}(\mathrm{NAsP})$ broad area laser devices lattice matched on $\mathrm{Si}(001)$ substrate is reported.

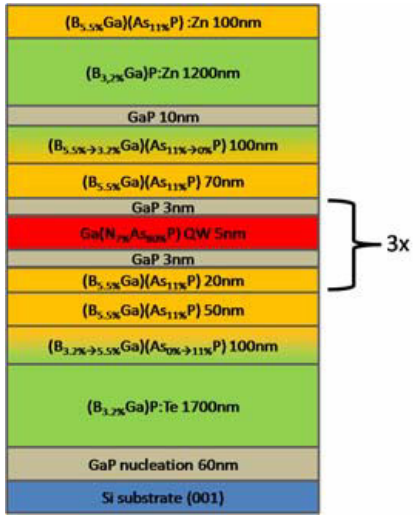

Fig. 1: Schematic structure of the Ga(NAsP)3QW laser on Si substrate.

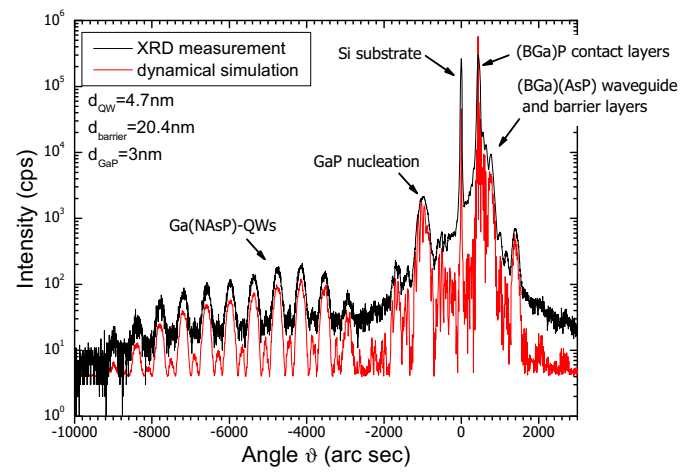

Fig. 3: High-resolution X-ray diffraction measurement of the $\mathrm{Ga}(\mathrm{NAsP})-3 \mathrm{QW}$ laser on $\mathrm{Si}$ around the (004)-reflection of $\mathrm{Si}$.

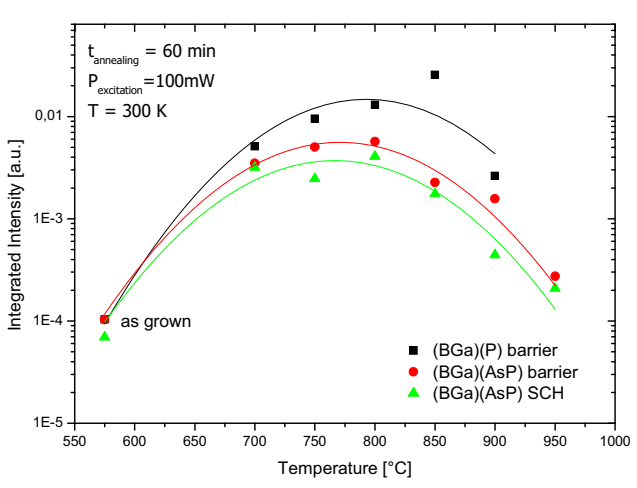

Fig. 2: Integrated PL-intensity at $300 \mathrm{~K}$ vs. annealing temperature for three different $\mathrm{Ga}(\mathrm{NAsP})$ multi-QW structures on $\mathrm{Si}(001)$.

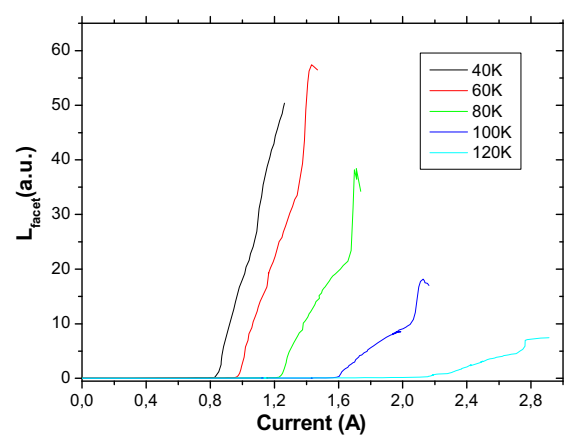

Fig. 4: Light-current curves from $40 \mathrm{~K}$ to $120 \mathrm{~K}$ for a $(50 \times 1000) \mu \mathrm{m} \mathrm{Ga}(\mathrm{NAsP})-3 \mathrm{QW}$ broad area laser.

\section{References:}

[1] B. Kunert et al., Journal of Crystal Growth 310, 4776 (2008)

[2] W. Shan et al., Phys. Rev. Lett. 82, 1221 (1999)

[3] B. Kunert et al., Appl. Phys. Lett. 88, 182108 (2006)

[4] B. Kunert et al., Elec. Lett. 42, 601 (2006)

[5] B. Kunert et al., Thin Solid Films 517, 140 (2008) 